\title{
Cellulose modification by recyclable swelling solvents
}

\author{
Ximing Zhang ${ }^{1}$, Tianjiao Qu², Nathan S. Mosier ${ }^{1}$, Lujia Han $^{2}$ and Weihua Xiao ${ }^{2^{*}}$ (1)
}

\begin{abstract}
Background: The invention of efficient systems for lignocellulose conversion is essential for economically feasible production of bio-based chemicals and biofuels. One limiting step is highly selective processes to quickly decrystallize the compact cellulose structure for efficient hydrolysis. We evaluated the impact of trifluoroacetic acid (TFA) and phosphorous acid (PA)-induced swelling of crystalline cellulose on enhancement of enzymatic digestion.

Results: In this study, two swelling agents, TFA and PA, are compared and found to be highly efficient for cellulose decrystallization at low temperatures within $1 \mathrm{~h}$. After treatment, the microfibril structure of swollen celluloses was observed to develop distinct microscopic morphology and subsequent enzymatic hydrolysis resulted over $90 \%$ cellulose conversion within $24 \mathrm{~h}$. The crystalline cellulose change was determined by reduction of loss of X-ray diffractability, and loss of resistance to enzymatic hydrolysis. NMR results suggest that both TFA and PA efficiently converted most of the crystalline cellulose regions to amorphous regions through cellulose chain relocation that inhibits recrystallization. It was found that the swelling mechanism is different between TFA and PA. To the best of our knowledge, it is the first time to compare and quantify the cellulose regions transformation by swelling agents.

Conclusion: This study shows the low-temperature swelling of different celluloses in TFA and PA reduces recalcitrance of crystalline cellulose to enzymatic hydrolysis. TFA and PA are both ideal candidate swelling agents for a closed system for ease of solvent recovery by either simple distillation or filtration. This study provides potentially useful agents in large-scale deconstruction of biomass.
\end{abstract}

Keywords: Swelling agent, Trifluoroacetic acid, Phosphoric acid, Cellulose modification, Crystallinity, Enzymatic hydrolysis

\section{Background}

Cellulose is the most abundant carbohydrate polymer on earth [1]. In recent decades, utilization of cellulose to produce biofuels, and functional molecules of interests have attracted numerous attention due to its renewable, carbon neutral, and environmental friendly features [14]. However, cellulose, a homopolymer of glucose, has a compact fibril structure possessing high crystallinity and extensive hydrogen bonds that are formed during biosynthesis which hinders the cellulose conversion efficiency

\footnotetext{
*Correspondence: xwhddd@163.com

${ }^{2}$ Laboratory of Biomass and Bioprocessing Engineering, College

of Engineering, China Agricultural University (East Campus), P.O. Box 191,

17 Qing-Hua-Dong-Lu, Haidian District, Beijing 100083, People's Republic

of China

Full list of author information is available at the end of the article
}

$[5,6]$. Pretreatment is required to make the compact cellulose structure accessible to enzyme or chemical catalyst to efficiently hydrolyze $\beta-1,4$ glycosidic bond $[7,8]$. Unlike pretreatments such as dilute acid, ammonia fiber expansion, ammonia recycle percolation pretreatment demanding high temperature or pressure, and cellulose solvent- and organic solvent-based lignocellulose fractionation (COSLIF) can fractionate lignocellulose components at modest reaction conditions [9]. The previous study shows that concentrated phosphoric acid (PA) is an effective cellulose solvent [9]. PA is a modest acid; under mild condition, cellulose can completely dissolve into it when its concentration is more than a critical value [10]. The cellulose dissolving phenomenon is known as solvent swelling effect. Similar as PA, swelling agent trifluoroacetic acid (TFA) attracts attention as it can effectively 
swell cellulose and be recycled due to its low boiling point, which provides an efficient and economical way to lower the cellulosic crystallinity and break up hydrogen bonds within the crystalline region [10-12].

Even at low temperatures, swelling agents can efficiently diffuse into the interstices between the fibrillary structural units of cellulose, as well as penetrate both ends into the elementary crystallites. This causes drastic changes in the crystal lattice structure [13]. As a result of swelling, cellulose crystallinity is significantly decreased, and the number and size of micro pores are increased, generating increased solvent-accessible surface area for hydrolyzing catalysts, such as enzymes $[14,15]$. When swollen cellulose is further heated, gelatinization occurs which results in dissolution of the cellulose into the liquid solvent, which is a transparent liquid. Dissolution completely disrupts the cellulose crystalline supramolecular structure. Rapid precipitation of this dissolved cellulose results in a disordered, amorphous cellulose. In our previous study, TFA was used comparing the effect of adding a gelatinization and precipitation step to enhance enzymatic hydrolysis of cellulose to reducing sugars. The data reported there showed that adding gelatinization/ precipitation does not further improve the cellulose reactivity from both enzymatic and chemical hydrolysis. The only significant effect was the small portion of cellulose that was hydrolyzed during gelatinization which could not be easily recovered by precipitation [15]. Our previous study determined that swelling cellulose is the key step for improving the cellulose reactivity and indicated that most of the changes relevant to enhancing hydrolysis occur through the swelling process.

To further understand the effect of swelling agents on cellulose substructure, we use two distinct celluloses in nature-cotton linters (Sigmacell, particle size ca $50 \mu \mathrm{m}$ ) and microcrystalline cellulose (Avicel PH 101, particle size ca $50 \mu \mathrm{m}$ ) as substrates. The Avicel PH 101 cellulose was prepared using sulfuric acid to hydrolyze poplar which is a representative of woody biomass and leave with more crystalline region cellulose; while the Sigmacell was prepared using herbaceous cotton linters cellulose contains both crystalline and amorphous regions cellulose. The cellulose in both untreated cotton linters and microcrystalline cellulose are in the form of cellulose I [16]. We report, for the first time, on comparisons between the physicochemical characterization of two types of cellulose by two swelling agents, TFA and PA. Both of them are known as swelling reagents for high efficiency for cellulose decrystallization which are used at high concentration and low temperature $\left(\mathrm{ca} 0{ }^{\circ} \mathrm{C}\right)[10$, 11]. However, based on our observation, the celluloses after swelling by TFA or PA have different morphological appearances before ethanol precipitation. PA results in a transparent cellulose gel in a short time, while TFA as solvent has the unique advantage in recovery as it is readily volatile. The difference in cellulose morphology indicates that there might be differences in the swelling mechanisms. Even though both agents have been reported for cellulose conversion, no previous studies focused on study and comparison their swelling mechanism at low temperature.

\section{Methods}

\section{Materials and reagents}

Sigmacell cellulose (Type 50, particle size $50 \mu \mathrm{m}$ ) and Avicel ${ }^{\circledR}$ PH 101 (particle size $50 \mu \mathrm{m}$ ) were purchased from Sigma-Aldrich. Trifluoroacetic acid (TFA, 99\%) and phosphoric acid (PA, 86.2\%) were purchased from Alfa Aesar. Ethanol (100\%) was purchased from Fisher Scientific (Houston, TX).

\section{TFA swelling of cellulose}

One gram of cellulose was mixed with $30 \mathrm{~mL}$ of TFA (99\%) in a $50 \mathrm{~mL}$ plastic Falcon tube. Then, the celluloseTFA mixture was incubated at $0{ }^{\circ} \mathrm{C}$ for a predetermined period. After swelling, approximately $60 \mathrm{~mL}$ of ethanol $(100 \%)$ was added with vigorous stirring, resulting in washed, swollen cellulose.

The cellulose was filtered through a Whatman GF/D filter paper and washed with an additional $30 \mathrm{~mL}$ ethanol $(80 \%)$ at a rate of $10 \mathrm{~mL}$ per addition to remove residual TFA. Deionized water was used to suspend the cellulose pellet which was then freeze-dried (LAGCONCO, Kansas City, MO). The TFA-swelled cellulose was collected and named as S-TFA (Sigmacell TFA-swelled) or A-TFA (Avicel-TFA-swelled) for further analysis.

\section{Phosphoric acid-swelled cellulose}

The phosphoric acid-swelling process was based on Zhang's [17] method with the minor modifications described below. One gram of cellulose and $3 \mathrm{~mL}$ distilled water were mixed in a $50 \mathrm{~mL}$ plastic Falcon tube, and then, $10 \mathrm{~mL} 0{ }^{\circ} \mathrm{C} \mathrm{PA}(86.2 \%)$ was added to the slurry with violent agitation. The resulting mixture was kept at $0{ }^{\circ} \mathrm{C}$ for a predetermined period with stirring every $10 \mathrm{~min}$. The cellulose was precipitated with ethanol, filtered, washed with additional ethanol, and then freeze-dried by the same procedure as TFA swelling. The phosphoric acid cellulose was collected and named as S-PA (Sigmacell PA swelled) or A-PA (Avicel PA swelled) for further analysis.

All six samples, untreated cellulose S-U (Sigmacell untreated) and A-U (Avicel untreated), TFA-treated cellulose (S-TFA and A-TFA), and PA-treated cellulose (S-PA and A-PA), share a similarly and extremely fine white powder appearance after freeze drying. 


\section{Physicochemical and morphological characterization Scanning electron microscopy (SEM)}

Scanning electron microscopy images were obtained via Hitachi S3400N (Japan) microscope at $15 \mathrm{kV}$ accelerating voltage. Prior to SEM observation, samples were placed in an ion sputter coater and subjected to gold sputtering for $0.5 \mathrm{~h}$. The surface morphology of cellulose samples randomly selected and captured at 500 magnifications.

\section{Atomic-force microscopy (AFM)}

Protocol of AFM experiment is previously reported by Chen et al. [18].

\section{$X$-ray diffraction (XRD)}

To determine the crystallinity of cellulose before and after TFA or PA treatment, samples were analyzed by X-ray diffraction (XRD). A LabX XRD-6000 (Shimadzu) diffract meter was used to generate pattern data with $\mathrm{Cu} \mathrm{K} \alpha$ radiation source $(\lambda=1.54060 \AA)$ over the angular range $2 \theta=5-40^{\circ}$ and a step degree of 0.04 . The crystalline index (CrI) was determined using the empirical method [10]:

$$
\mathrm{CrI}=\frac{I_{002}-I_{\mathrm{am}}}{I_{002}} \times 100
$$

where $I_{002}$ is the maximum intensity of 002 peak at approximately $2 \theta=22^{\circ}$, while $I_{\mathrm{am}}$ is the amorphous diffraction intensity at approximately $2 \theta=18.0^{\circ}$.

\section{Fourier transform infrared (FTIR) spectroscopy}

The functional groups' change of swelled cellulose was analyzed by FTIR. The spectra were acquired on a Perkin Elmer Spotlight 400 FTIR spectrometer (Perkin Elmer, Waltham, MA, USA) working in a transmittance mode. Before scanning, the sample pellets were prepared by mixing with spectroscopic grade $\mathrm{KBr}$ in a ratio of $1: 100$ $(\mathrm{w} / \mathrm{w})$ and then pressed under vacuum. FTIR spectra were recorded at $4 \mathrm{~cm}^{-1}$ resolution and 64 scans per sample in the range of $4000-500 \mathrm{~cm}^{-1}$.

\section{Solid-state CP/MAS ${ }^{13} \mathrm{CNMR}$}

Solid-state ${ }^{13} \mathrm{C}$ CP/MAS NMR were performed at $100 \mathrm{MHz}$ resonance frequency on a Bruker Avance
DPX400 NMR spectrometer with a $4 \mathrm{~mm}$ CP/MAS probe and samples spinning at $5 \mathrm{kHz}$ MAS speed. ${ }^{13} \mathrm{C}$ $\mathrm{CP} / \mathrm{MAS}$ NMR were conducted with 3 ms contact time, $30 \mathrm{~ms}$ acquisition time, $2 \mathrm{~s}$ recycle delays, and in 1600 scans. The data were analyzed by MestReNova and Origin software.

\section{Cellulose reducing-end determination}

The molar concentration of reducing ends of the cellulose was measured by the modified BCA method [19]. BCA reaction solution A was prepared by dissolving $0.971 \mathrm{~g}$ of disodium 2,2'-bicinchoninate, 27.14 $\mathrm{g}$ of $\mathrm{Na}_{2} \mathrm{CO}_{3}$, and $12.1 \mathrm{~g}$ of $\mathrm{NaHCO}_{3}$ dissolved in $500 \mathrm{~mL}$ of distilled water. Solution B contained $0.624 \mathrm{~g}$ of $\mathrm{CuSO}_{4} \cdot 5 \mathrm{H}_{2} \mathrm{O}$ and $0.631 \mathrm{~g}$ of L-serine dissolved in $500 \mathrm{~mL}$ of water. Equal volumes of solution A and solution B were mixed to form the working solution before use. One milliliter of BCA working solution was mixed with $1 \mathrm{~mL}$ sample solution containing cellulose $(5 \mathrm{mg} / \mathrm{mL})$ in a tube with stopper. Glucose solutions with concentrations from 0 to $50 \mu \mathrm{M}$ were used as standards. The mixture was incubated for $30 \mathrm{~min}$ at $75{ }^{\circ} \mathrm{C}$ in a convection oven. For cellulose sample, the tubes were vortex-mixed every $10 \mathrm{~min}$ to increase the interaction between cellulose and working solution. The tubes were rapidly cooled to $20^{\circ} \mathrm{C}$ using tap water. After cooling, reaction mixtures were transferred to a centrifuge tube and centrifuged for $5 \mathrm{~min}$ at a speed of $10,000 \mathrm{~g}$. The supernatant was measured at $560 \mathrm{~nm}$ on a UV-Vis spectrometer.

\section{Enzymatic hydrolysis}

To evaluate the enzymatic digestibility of swelled cellulose, enzymatic hydrolysis experiments were performed in $50 \mathrm{~mL}$ plastic round-bottom Falcon tubes in triplicate in a thermostatically controlled shaker at $50{ }^{\circ} \mathrm{C}$ and $250 \mathrm{rpm}$. The initial solid loading of $1 \%(\mathrm{w} / \mathrm{v})$ was added into $5 \mathrm{~mL}, 50 \mathrm{mM}$ sodium citrate buffer where $\mathrm{pH}$ was maintained around 4.8. Cellic ${ }^{\mathrm{TM}} \mathrm{Ctec} 2$ (aggressive cellulase and a high level of $\beta$-glucosidase, Novozymes North America, Inc., Franklinton, NC), at an enzyme loading of 7.0 FPU/g cellulose, was used for treated and untreated samples. Sodium azide $(0.2 \%, \mathrm{w} / \mathrm{v})$ was added to inhibit microbial growth during hydrolysis. Samples were taken at predetermined intervals and immediately filtered through $0.2 \mu \mathrm{m}$ nominal-pore-size nylon syringe filter (Pall, Port Washington, NY) to remove the residual substrate. Filtrate was then stored at $-20{ }^{\circ} \mathrm{C}$ until analysis by HPLC.

The glucose yield was calculated as:

$$
\text { Glucose yield }(\%)=\frac{\text { concentration of glucose } \times \text { volume of reaction }}{\text { total mass of cellulose }} \times 100 \text {. }
$$


Table $1 \mathrm{Crl}$ of treated and untreated cellulose

\begin{tabular}{lll}
\hline & Sigmacell & Avicel \\
\hline Untreated & 81.1 & 83.0 \\
TFA & 42.1 & 37.6 \\
PA & 25.3 & 29.0 \\
\hline
\end{tabular}

The concentration of glucose was quantified by HPLC using an HPX-87H AMINEX ion exchange column (BioRAD, Hercules, CA), a Waters 1525 pump, and a Waters 2412 Refractive Index detector (Waters, Milford, MA). The data were stored and processed using Empower ${ }^{\mathrm{TM}} 2$ Chromatography Data Software (Waters Corp., Milford, MA). The mobile phase was $5 \mathrm{mM}$ sulfuric acid in distilled, deionized water at flow rate of $0.6 \mathrm{~mL} / \mathrm{min}$. The column temperature was maintained at $60{ }^{\circ} \mathrm{C}$. The injection volume was of $50 \mu \mathrm{L}$. The concentrations of glucose in the aqueous phase were determined using external calibration standards.

\section{Results and discussion}

Analysis of cellulose crystalline content by X-ray diffraction and FTIR spectroscopy

Crystallinity change as a function of increasing swelling time $(0,1,12,24$, and $48 \mathrm{~h})$ for both swelling agents was measured. For both PA and TFA, CrI decreased rapidly within $1 \mathrm{~h}$ at $0{ }^{\circ} \mathrm{C}$ and reached a stable $\mathrm{CrI}$ value (Table 1 ). For quantification purpose, $1 \mathrm{~h}$ was selected as swelling treatment time for fully swollen cellulose to compare the effect on cellulose substructural change. Taking into consideration of the economic feasibility and energy input for large-scale applications, temperatures lower than $0{ }^{\circ} \mathrm{C}$ were not considered as influencing factors in this study. However, temperatures of -15 and $-20{ }^{\circ} \mathrm{C}$ (lower than the freezing point of TFA) were tested as a comparison with $0{ }^{\circ} \mathrm{C}$ in previously reported work [15]. The crystallinity indices (CrIs) reported that there were very close to our results obtained at $0{ }^{\circ} \mathrm{C}$. Therefore, we used $0{ }^{\circ} \mathrm{C}$ as the standard swelling temperature for both TFA and PA in this work. The CrIs of the untreated Sigmacell and Avicel cellulose were 81 and $83 \%$, respectively. TFA-treated Sigmacell and Avicel cellulose stabilized with $\mathrm{CrI}$ values of 42 and 38\%, while PA-treated Sigmacell and Avicel were 25 and $29 \%$, respectively. The trend of CrI changes suggests that PA disrupts cellulose crystallinity more efficiently than TFA.

By further analyzing the XRD spectra shown in Fig. 1, it further confirms that loss of crystallinity during swelling results in cellulose crystal transmutation. The untreated celluloses were in cellulose I polymorph patterns. From the spectra, both cellulose $I_{\alpha}$ and cellulose $I_{\beta}$ coexist in the swelled samples. As shown with the

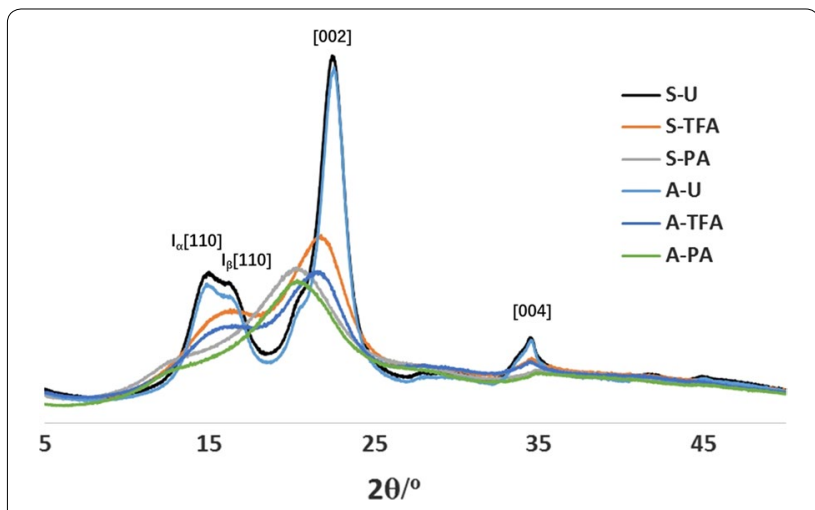

Fig. 1 Normalized XRD diffractograms of untreated and treated cellulose

designated peaks, $2 \theta=16^{\circ}$ represents the cellulose $I_{\alpha}[1$ $10]$ lattice plane, and $18^{\circ}$ represent cellulose $I_{\beta}\left[\begin{array}{lll}1 & 1 & 0\end{array}\right]$ lattice plane, $22.7^{\circ}$, and finally, $35^{\circ}$ corresponds to the $\left[\begin{array}{lll}0 & 0 & 2\end{array}\right]$ and [ [ $\left.\begin{array}{lll}0 & 0 & 4\end{array}\right]$ lattice planes. After the swelling process, TFA- and PA-treated celluloses had distinct spectra shifts, indicating different forms of cellulose crystal transformations.

For the TFA-treated celluloses, the cellulose $I_{\alpha}[1$ $10]$ lattice plane was shifted from $2 \theta$ value of $16^{\circ}$ to a higher $2 \theta$ value, merging with cellulose $I_{\beta} 2 \theta$ value. The intensity for [ [ $\left.\begin{array}{lll}0 & 0 & 2\end{array}\right]$ lattice plane was significantly decreased and shifted angle to a lower $2 \theta$ value. For the PA-treated celluloses, the intensity for [ $\left[\begin{array}{lll}0 & 0 & 2\end{array}\right]$ lattice plane was further weakened compared to TFA-treated celluloses and shifted to a lower $2 \theta$ value. It is noticed that cellulose $I_{\alpha}$ and $I_{\beta}\left[\begin{array}{lll}1 & 1 & 0\end{array}\right]$ plane lattice signals were not detected, while [ $\left[\begin{array}{lll}1 & 1 & 0\end{array}\right]$ lattice plane with $2 \theta$ value $12.2^{\circ}$ was observed for both A-PA and S-PA. TFAtreated celluloses did not have $2 \theta$ values for $\left[\begin{array}{lll}1 & 1 & 0\end{array}\right]$ that corresponded to PA-treated celluloses. Both PA- and TFA-treated spectra show the transition of cellulose I to cellulose II. PA treatment resulted in a $2 \theta$ value of $12^{\circ}$, representing the $\left[\begin{array}{lll}1 & 1 & 0\end{array}\right]$ lattice plane of cellulose II. TFA treatment resulted in a cellulose I $\left[\begin{array}{lll}1 & 1 & 0\end{array}\right]$ increased angle shift from $16.5^{\circ}$ to close to [2 00 [ [ $\left.20-23\right]$.

These data show that the cellulose I structure was disrupted and resulted in more disorder cellulose structure [24]. According to our previous study, cellulose II was formed by recrystallization of swollen cellulose when freeze-dried [15]. However, distinct spectra patterns between TFA- and PA-treated cellulose suggest that, besides recrystallization, there may be different effects on the crystal structure between the two swelling agents.

To further examine the changes to the cellulose structure, FTIR analyses of the samples were performed (Fig. 2 and Table 2). Related to the crystallinity change 


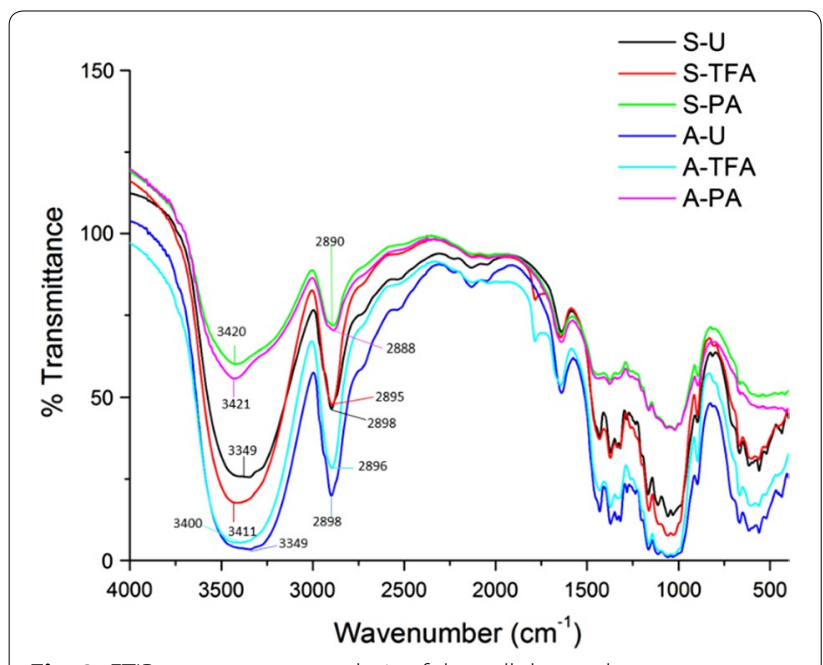

Fig. 2 FTIR spectroscopy analysis of the cellulose substrates

Table 2 FTIR peaks' wavenumber and absorbance

\begin{tabular}{lll}
\hline S-U & $3349.60 \mathrm{~cm}^{-1} / 0.59 \mathrm{~A}$ & $2898.34 \mathrm{~cm}^{-1} / 0.33 \mathrm{~A}$ \\
S-TFA & $3411.22 \mathrm{~cm}^{-1} / 0.75 \mathrm{~A}$ & $2895.08 \mathrm{~cm}^{-1} / 0.32 \mathrm{~A}$ \\
S-PA & $3420.61 \mathrm{~cm}^{-1} / 0.22 \mathrm{~A}$ & $2890.04 \mathrm{~cm}^{-1} / 0.14 \mathrm{~A}$ \\
A-U & $3349.19 \mathrm{~cm}^{-1} / 1.46 \mathrm{~A}$ & $2898.81 \mathrm{~cm}^{-1} / 0.70 \mathrm{~A}$ \\
A-TFA & $3400.10 \mathrm{~cm}^{-1} / 1.26 \mathrm{~A}$ & $2896.17 \mathrm{~cm}^{-1} / 0.54 \mathrm{~A}$ \\
A-PA & $3421.90 \mathrm{~cm}^{-1} / 0.25 \mathrm{~A}$ & $2888.99 \mathrm{~cm}^{-1} / 0.15 \mathrm{~A}$ \\
\hline
\end{tabular}

before and after the swelling process, $\mathrm{O}-\mathrm{H}$ stretching and $\mathrm{C}-\mathrm{H}$ stretching were the two most significant signals that can be observed in the FTIR spectra.

The $\mathrm{O}-\mathrm{H}$ stretching is observed at the absorbance of $3600-3100 \mathrm{~cm}^{-1}$ which includes valence vibration of hydrogen-bonded $\mathrm{OH}$ groups, i.e., the intramolecular hydrogen bond, intermolecular hydrogen bond, and the $\mathrm{O}-\mathrm{H}$ stretching. The signal intensities are significantly weakened by PA swelling, while TFA swelling does not result in as significant change as PA does. The $\mathrm{O}-\mathrm{H}$ stretching corresponds both to the larger CrI decrease by PA than by TFA and to the XRD spectra results. Both TFA and PA can significantly decrease the cellulose structure crystallinity, while decrystallization by PA was more complete than by TFA.

The $\mathrm{C}-\mathrm{H}$ stretching signal indicates a high percentage of long-chain hydrocarbons in the sample. Decreasing signal for $\mathrm{C}-\mathrm{H}$ stretching $\left(2895 \mathrm{~cm}^{-1}\right)$ was observed after the swelling process in both substrates. PA decreased more than TFA. This is consistent with what was observed in the XRD results.

The drastically decrease of crystallinity of cellulose and lignocellulosic substrates to more amorphous cellulose regions was also observed by treating cellulose residue using ionic liquids, confirming that both swelling agents and ionic liquids could enhance the cellulose conversion efficiency by breaking the cellulose microstructure hydrogen bond to increase the accessible area [24].

\section{Study redistribution and recrystallization of cellulose content by $\mathrm{CP} / \mathrm{MAS}{ }^{13} \mathrm{C}$ NMR}

In addition to XRD and FTIR, CP/MAS ${ }^{13} \mathrm{C}$ NMR provided insight into cellulosic substructural changes from swelling treatment. Larsson et al. [25] and Wickholm et al. [26] determined spectral fitting methods and assignment for cellulose using cotton linter and several other cellulose sources. They found that the deconvoluted NMR signal of C-4 region (80-92 ppm) gives key information on the specific cellulose fibril structure. The C-4 region divided into two distinct region ranges: crystalline region and amorphous region [27]. Region from 86 to $92 \mathrm{ppm}$ represents cellulose in more ordered forms. It is crystalline and defined separately as $I_{\alpha}$ at $89.35 \mathrm{ppm}$, $I_{(\alpha+\beta)}$ at $88.5 \mathrm{ppm}$, and $I_{\beta}$ at $87.91 \mathrm{ppm}$. A fourth form of cellulose with more mobility was determined and named as paracrystalline at $88.25 \mathrm{ppm}$. It was in-core or beneath fibril with less-ordered crystalline form compared to the others. All of the four crystalline regions are surface inaccessible to the surrounding solvent.

Region from 80 to $86 \mathrm{ppm}$ represents more disordered forms or amorphous cellulose. They are defined as (solvent) accessible fibril surface, (solvent) inaccessible fibril surface and cellulose oligomers, respectively [26]. Solvent-accessible fibril surface at 84.95 and $83.26 \mathrm{ppm}$ is determined to be amorphous cellulose at fibril or crystallite surfaces accessible to surrounding solvent [28, 29]. In contrast, inaccessible fibril surface at $83.43 \mathrm{ppm}$ are defined as amorphous form cellulose portion which is inaccessible to the surrounding solvent. Cellulose oligomers at $81.68 \mathrm{ppm}$ are defined as less-ordered forms of carbohydrates or hemicellulose residues [25].

From Fig. 3 and Table 3, the NMR spectra showed that untreated celluloses had a dominant crystalline peak compared to the amorphous region. After TFA swelling process, the amorphous region was significantly enhanced, while the crystalline region decreased. The peaks change trends correspond to the CrI decrease after TFA swelling treatment. After PA treatment, more severe decrease of the crystalline region could be observed. The dominant peak fell on amorphous region. With the transition of the crystalline peaks to the amorphous peaks, the CrI value of both PA-treated sample decreased more substantial than the TFA-treated sample (Table 1). The $\mathrm{CrI}$ value changes indicate the $\mathrm{H}$-bonding rearrangement undergoing within the cellulose structure for both TFA-treated and PA-treated sample. The PA treatment has more thoroughly change in the cellulose substructure 


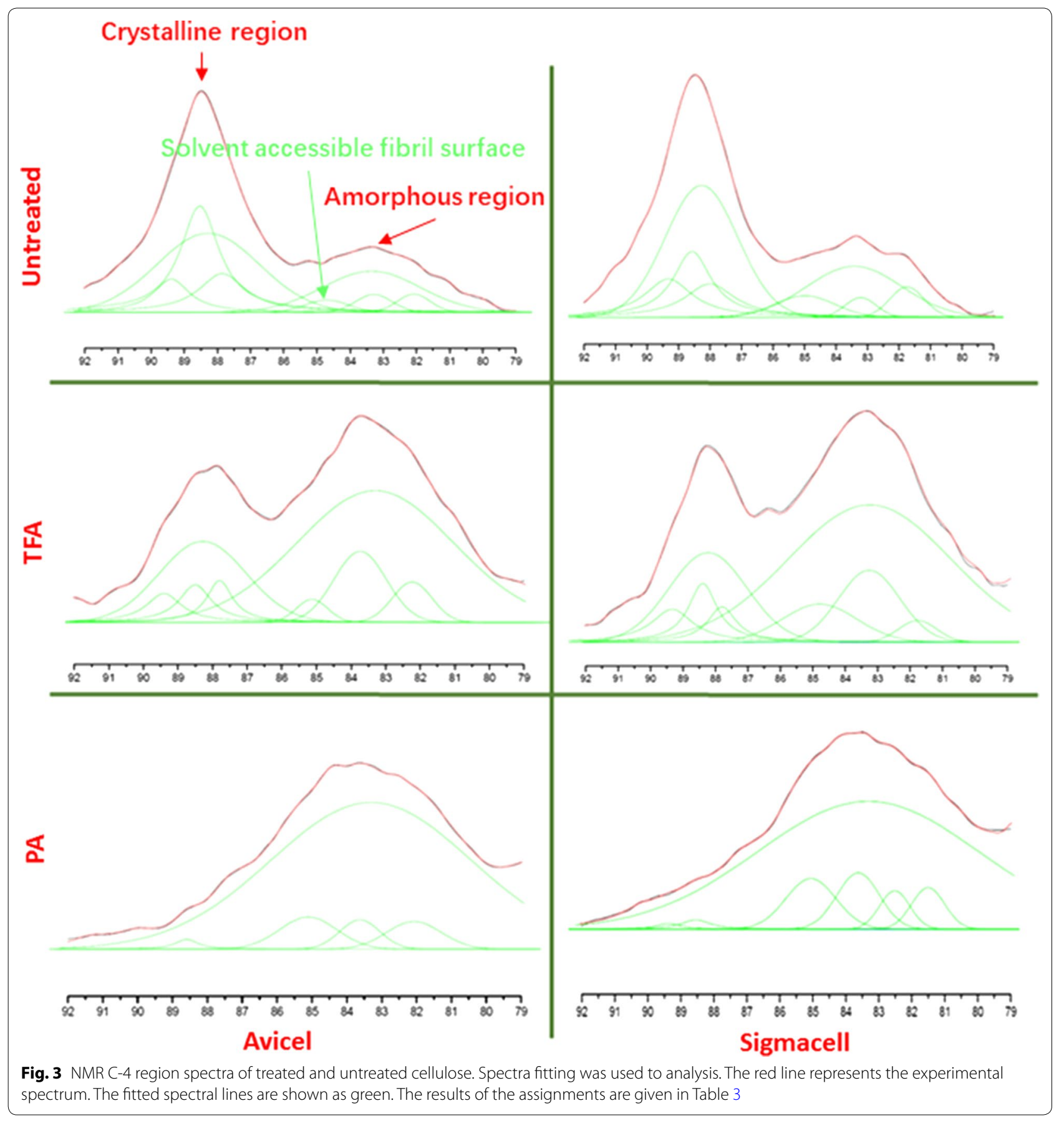

than TFA swelling treatment, which can be further confirmed and analyzed by the deconvoluted peaks.

Deconvoluted peaks (green line) show the details of assignment and spectra fitting of CP-MAS NMR signal in the C-4 region before and after swelling (Table 3 and Fig. 3). As the crystalline and amorphous region signals are complementary, the deconvoluted peak signals are integrated with total intensity of $100 \%$. Figure 4 compares relative signal intensities using the deconvoluted peak results of Fig. 3. Untreated cotton linter has a large percentage which includes crystalline and paracrystalline portion. For the amorphous portion, the inaccessible fibril surfaces are larger than accessible fibril surfaces. After TFA treatment, the accessible fibril surface was dramatically increased by $50.6 \%$ with the inaccessible fibril surface portion and crystalline and paracrystalline 
Table 3 Assignments of signals in the C-4 region of the untreated and treated cellulose spectra

\begin{tabular}{lll}
\hline & Chemical shift (ppm) & Line shape \\
\hline$I_{a}$ & $89.35(0.03)^{\mathrm{a}}$ & Lorentz \\
$I_{(a+\beta)}$ & $88.50(0.10)$ & Lorentz \\
Paracrystalline & $88.25(0.03)$ & Gauss \\
$I_{\beta}$ & $87.91(0.15)$ & Lorentz \\
Accessible fibril surfaces & $84.95(0.14)$ & Gauss \\
Inaccessible fibril surfaces & $83.43(0.18)$ & Gauss \\
Accessible fibril surfaces & $83.26(0.06)$ & Gauss \\
Cellulose oligomers & $81.68(0.15)$ & Gauss \\
\hline
\end{tabular}

${ }^{a}$ Values in brackets are standard errors

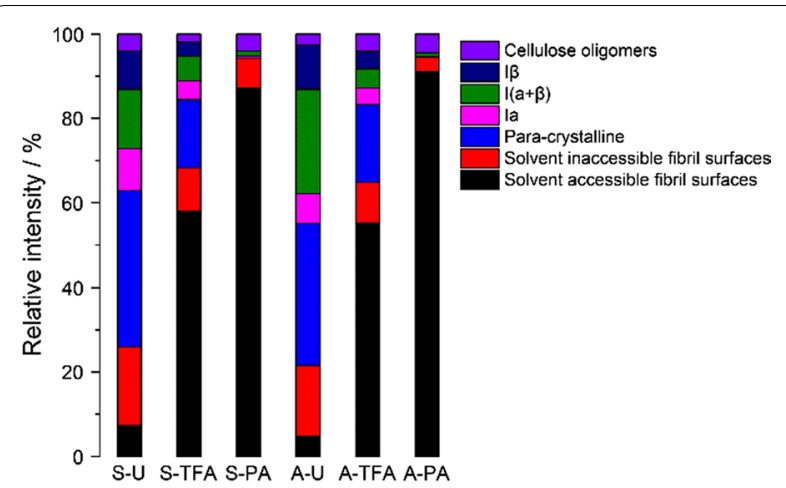

Fig. 4 Relative signal intensities obtained by fitting of C-4 region spectra of untreated and treated cellulose

regions decreased, indicating the disruption of rigidly crystal cellulose construction into amorphous one. PA treatment further increased the accessible region while maintaining similar inaccessible fibril surface fraction. However, accessible fibril surfaces increase to $87.2 \%$. Most of the crystalline and paracrystalline regions declined to approximately $2 \%$ in total. It is worth noting that the relative intensities among crystalline region $I_{\alpha}$, $I_{(\alpha+\beta)}$, and $I_{\beta}$ was not significantly changed. This is likely due to the low temperature of the swelling treatment, as solid-state conversion of $I_{\alpha}$ to $I_{\beta}$ requires high temperature for transforming the crystals [30]. Interestingly, when Avicel is used as cellulose substrate, a similar transition is observed.

Overall, the inner structure of cellulose was disrupted after being treated by TFA, whereas PA treatment can further improve accessible fibril surfaces region into dominant percentage.

\section{Analysis of crystalline cellulose change by microscopy}

Scanning electron microscopy was used to evaluate changes in surface morphology of cellulose substrate before and after swelling. Figure 5 shows the SEM images at $500 \times$ magnification. Untreated cellulose particles are generally rod-shaped and with orderly arranged fibers observable on the cellulose surface.

After swelling with TFA, a significant morphology change was observed compared to untreated cellulose substrates. The surface of the particles was more irregular with some fibers visible protruding from the surface. This confirms the previous results which showed that the TFA dimer at low temperature can penetrate the cellulose structure to swell the cellulose [11].

The phosphoric acid treatment did not generate the same morphological surface change as TFA treatment. The particles were distributed loosely after PA swelling, while the surface of the particles was similar to the untreated cellulose. This is in contrast to the XRD and FTIR data which show greater changes during PA treatment.

Different swelling mechanisms between TFA and PA were also observed by atomic-force microscopy (AMF). Figure 6 shows the AMF images of the native and swollen cellulose substrates. Untreated cellulose has longitudinal and rod-shaped particles. Untreated microcrystalline cellulose (Avicel) has a larger diameter compared to cotton linters. After the TFA treatment, the length of microcrystalline cellulose was dramatically reduced to shorter fractions and loosely distributed with a more spherical shape due to TFA swelling. Sigmacell cotton linters with native amorphous regions provide unique advantage for the TFA molecules penetration and generate large sphere interconnected swollen cellulose. The length of the TFAtreated cotton linters does not change significantly.

The PA-swollen Avicel and Sigmacell celluloses were observed with cellulose redistribution and recrystallization with thinner-shaped rod. Celluloses are more tightly packed compared to loosely distributed TFA-treated celluloses. The AFM images also show that swollen effect of PA is not as obvious as TFA, in contrast to the XRD and FTIR data which show the opposite.

\section{Rates of enzymatic hydrolysis of swollen cellulose}

The analyses described above show significant decreases in cellulose crystallinity and increases in solvent-accessible cellulose surface, both of which correlate strongly with cellulose digestibility by enzymes [31, 32]. We have confirmed this by performing enzymatic hydrolysis of the untreated, TFA-treated, and PA-treated cellulose samples (Fig. 7). Prior to swelling, the saccharification rates and yields of both varieties of cellulose are low (ca 40\%) through $24 \mathrm{~h}$ of reaction. The results are consistent with the previous reports and indicate that cellulose is partially accessible due to their compact crystalline structure [10]. The untreated Sigmacell yields 6\% more glucose 


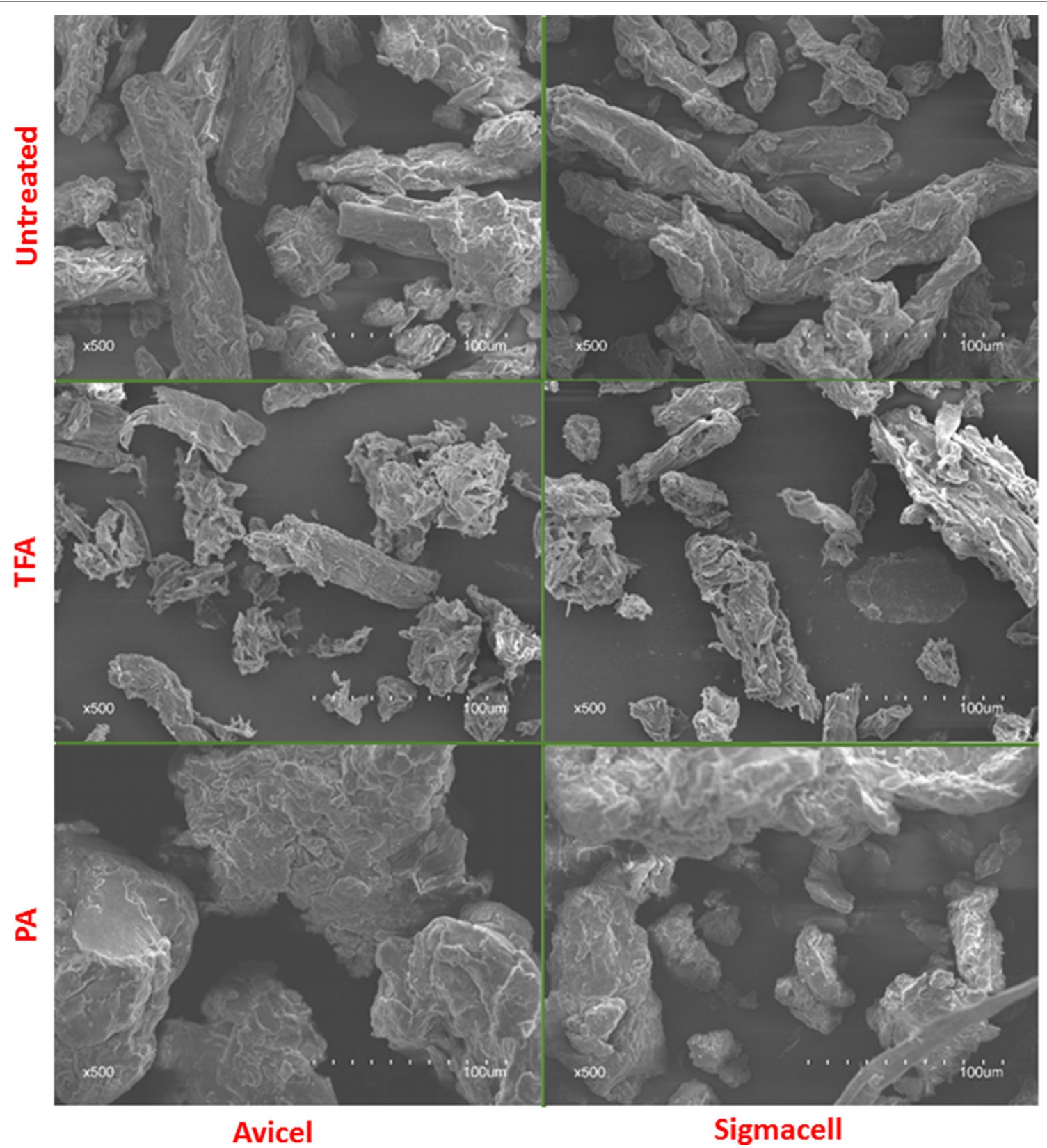

Fig. 5 Scanning electron microscopy (SEM) images of native and swollen substrates

compared to Avicel, which might be due to the amorphous regions of cotton linters compared to high crystallinity of Avicel.

After treatment by PA or TFA, the enzymatic hydrolysis yields were dramatically improved for both varieties of cellulose, likely due to the reduced crystallinity. PA-treated cellulose has a higher initial kinetic rate (0-3 h) compared to TFA-treated cellulose. This corresponds to the higher percentage of solvent-accessible fibril surfaces in PA-treated celluloses compared to TFA-treated celluloses. Interestingly, PA-swelled Sigmacell has a higher glucose yield compared to PAswelled Avicel, while maintaining a similar initial hydrolysis rate. This can be interpreted that, even after swelling, the microcrystalline Avicel that is manufactured by sulfuric acid hydrolysis of wood pulp retains some of its recalcitrance after PA swelling. 

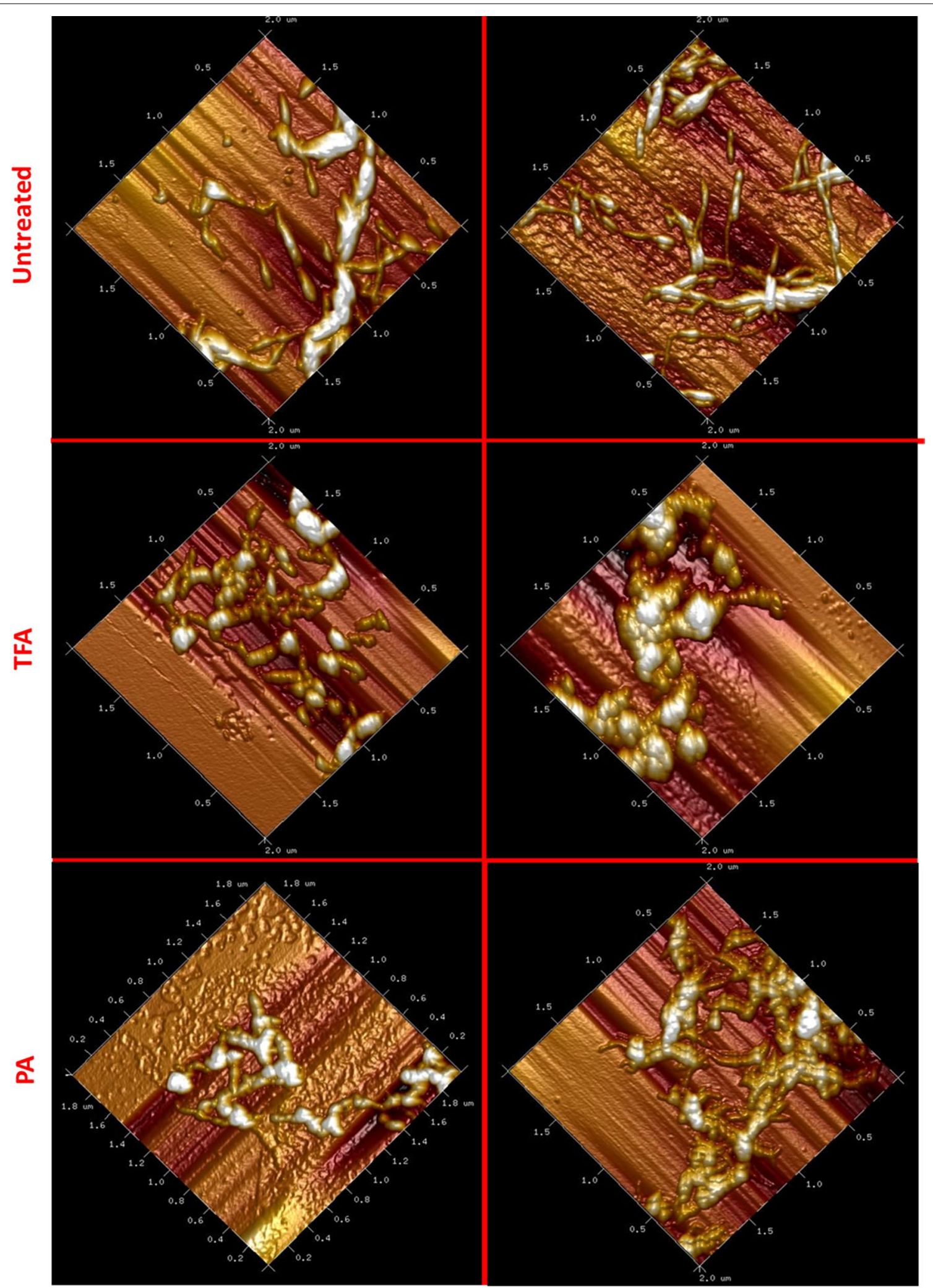

Avicel

Sigmacell

Fig. 6 AFM analysis of native and swollen substrates 


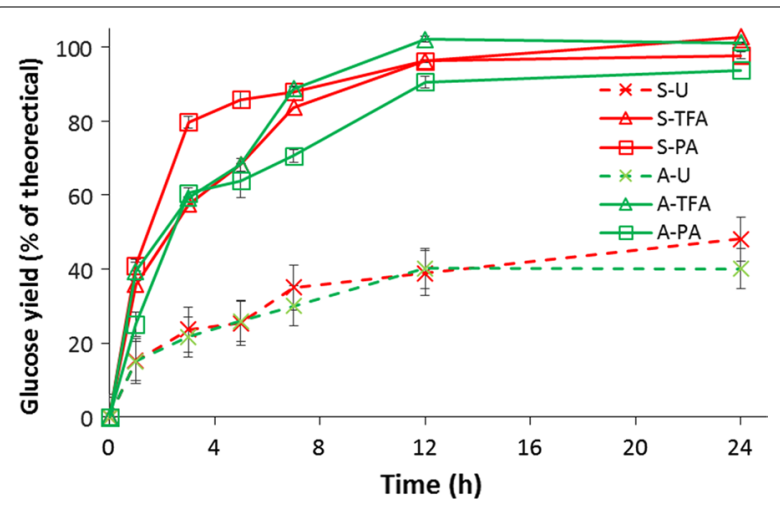

Fig. 7 Rates of enzymatic hydrolysis of untreated and swelled cellulose with Cellic ${ }^{\circledR}$ Ctec2 (Novozymes) loading 7 FPU/g glucan

Compared to PA-treated cellulose, TFA-treated Avicel and Sigmacell do not have a significant difference both on trend of glucose yield and reaction rates. Both of the TFA-treated celluloses were fully converted into glucose by $24 \mathrm{~h}$. However, the slower rates during the initial hydrolysis for TFA-treated cellulose correlated to the lower fraction of fibril that was solvent accessible compared to PA-treated celluloses. As the surface of the TFA-treated cellulose was hydrolyzed, the newly exposed paracrystalline and cellulose oligomers were quickly digested.

\section{Analyses of swelling agent effect on celluloses}

Low-temperature swelling by TFA and PA results in efficient disruption of cellulose crystallinity. TFA is recommended as it has a wide operational condition $(-20$ to $78{ }^{\circ} \mathrm{C}$ ) and distillation will be feasible after the swelling process. Compared to TFA, PA is unique that during swelling process, cellulose becomes a gel in a short time. PA may be also recyclable after the swelling process.

$\mathrm{X}$-ray diffraction results showed that, after swelling, CrI indices were decreased significantly for both swelling agents. PA has a larger decrease in CrI. More importantly, XRD spectra shifts showed that PA and TFA may have different swelling mechanisms. The SEM and AFM results showed different morphological changes after the TFA (flaky on the surface of the swelled cellulose), while PA treatment does not generate as significant change as the surface of TFA-swelled cellulose. Furthermore, AFM showed that TFA treatment results in predominantly spherical particles, while PA generated cylindrical morphology. The FTIR results confirmed that the intramolecular bonding was changed during treatment. The different effects and extent of cellulose microstructure changes are determined and analyzed by deconvolution of NMR data. The results clearly showed that choice of
Table 4 Reducing ends of the untreated and treated cellulose

\begin{tabular}{lll}
\hline Sample & $\begin{array}{l}\text { Reducing sugar concentration } \\
(\boldsymbol{\mu} \boldsymbol{M})\end{array}$ & $\begin{array}{l}\text { Standard } \\
\text { deviation }\end{array}$ \\
\hline A-U & 11.2 & 0.3 \\
A-TFA & 47.6 & 2.1 \\
A-PA & 9.8 & 1.2 \\
S-U & 12.3 & 0.9 \\
S-TFA & 55.3 & 5.3 \\
S-PA & 10.2 & 0.8 \\
\hline
\end{tabular}

swelling agent is more important than cellulose source for determining the type and extent of changes to the cellulose.

Enzymatic hydrolysis is the most effective way to evaluate the resulting change in cellulose reactivity after swelling. We found that the early stage $(<6 \mathrm{~h})$ hydrolysis of phosphoric acid-swelled cellulose has significantly higher initial hydrolysis rates compared to TFA. Regardless of cellulose type, phosphoric acid can convert more of the cellulose into accessible fibril surface, with 29\% higher accessible surface than TFA. This might explain why the phosphoric acid treated cellulose has faster initial hydrolysis rates than TFA-treated cellulose. Combined with the results of sugar reducing ends after swelling process, as shown in Table 4, TFA has a more significant effect on exposing increased sugar reducing ends at low temperature, while PA mainly physically relocates the cellulose from largely solvent inaccessible to more solvent accessible. This might be explained by the fact that concentrated PA has a freezing point slightly below $4{ }^{\circ} \mathrm{C}$. TFA has less effect on changing cellulose crystal forms, but has a higher impact on decreasing the degree of polymerization of cellulose.

Taken together, these data give us insights into different mechanisms for TFA and phosphoric acid-swelling process. CrI change of cellulose is not the only indicator on cellulose reactivity, and more importantly, cellulose substructure change should be taken into consideration, since swelling agents function differently.

Both TFA and PA treatments resulted in a significant cellulose decrystallization. We used CP/MAS ${ }^{13} \mathrm{C}$ NMR as a non-destructive tool to further quantify changes to the cellulose super-molecular structure upon TFA and PA treatment [26]. We found the spectral fitting of NMR spectrum of cellulose I signal cluster between 80 and $92 \mathrm{ppm}$ which have significant different strength between the TFA- and PA-treated cotton linters and microcrystalline cellulose [26]. The NMR results confirmed higher solvent-accessible fiber region of PA treatment cellulose correlate to higher initial hydrolysis 
rates for cellulose by enzymes. TFA caused significant changes to the microscopic morphology of the cellulose character compared to PA-treated cellulose, TFAtreated cellulose presented a lower percent of the total portion that was solvent accessible but has a much higher amount of reducing sugar ends, suggesting partial hydrolysis of the cellulose during treatment.

To sum up, swelling mechanisms between PA and TFA lie in three ways. First, morphologically, PA generates spherical swollen cellulose, TFA generates cylindric swollen cellulose. The morphology of the swollen celluloses indicates that PA results in a more thoroughly swelling change in cellulosic substructure as more space was created in the sample (confirmed by XRD results). Second, chemically, the PA is more efficient in creating inner space by breaking inter cellulose chain hydrogen bonds than hydrolyzing 1,4-glycosidic bonds in cellulose chain, while TFA functions as dimer to create more porous cellulosic structure under low temperature, as well as generated much higher sugar reducing ends content than PA. Third, effects on enzymatic hydrolysis conversion efficiency of two swelling agents demonstrated that creating cellulose inner space between cellulose chain is more important than creating sugar reducing ends for anchoring biocatalyst active site.

\section{Conclusions}

The results demonstrated that TFA and PA have different mechanisms on cellulose modification, but result in similar yields during enzymatic hydrolysis after $24 \mathrm{~h}$. Hydrolysis data showed that cellulose after TFA treatment has more homogeneity as TFA catalyzed and exposed cellulose with sugar reducing ends. PA treatment has more impact on cellulose internal fibril structure at low temperature, but does result in more cellulose chain ends as determined by exposed reducing sugars. The swelling process demonstrated that crystallinity change is not the only indicator for cellulose modification and reactivity improvement, while cellulose substructure change is more important.

\section{Authors' contributions \\ $\mathrm{XMZ}$ and TJQ designed and performed experiments on conditions for gener- ating amorphous cellulose and comparison of TFA- and PA-swollen celluloses through different characterization method. WHX, NSM, and LJH assisted in design and supervision of the experiments and edited the manuscript. All authors read and approved the final manuscript.}

\footnotetext{
Author details

${ }^{1}$ Laboratory of Renewable Resources Engineering, Department of Agricultural and Biological Engineering, Purdue University, West Lafayette, IN 47907, USA. ${ }^{2}$ Laboratory of Biomass and Bioprocessing Engineering, College of Engineering, China Agricultural University (East Campus), P.O. Box 191, 17 Qing-Hua-Dong-Lu, Haidian District, Beijing 100083, People's Republic of China.
}

\section{Acknowledgements}

The authors are extremely grateful to the financial support by the National Key R\&D Program of China (2016YFE0112800) and National Natural Science Foundation of China (31671572).

\section{Competing interests}

The authors declare that they have no competing interests.

\section{Availability of data and materials}

All data generated or analyzed during this study are included in this published article.

\section{Consent for publication \\ Not applicable.}

\section{Ethics approval and consent to participate}

Not applicable.

\section{Funding}

This research was financially supported by the National Key R\&D Program of China (2016YFE0112800), National Natural Science Foundation of China (31671572), and National Higher Education Institution General Research and Development Funding (2018TC027).

\section{Publisher's Note}

Springer Nature remains neutral with regard to jurisdictional claims in published maps and institutional affiliations.

Received: 27 April 2018 Accepted: 5 July 2018

Published online: 13 July 2018

References

1. Zhang $X, X u$ J, Pandey $P$, Cheng J. Pretreatment of corn stover for enzymatic saccharification improvement by using the combination of alkaline reagents at mild temperatures. In: ASABE 2012 Dallas, Texas, July 29-Aug 1, 2012; 2012

2. $X u$ J, Zhang $X$, Cheng JJ. Pretreatment of corn stover for sugar production with switchgrass-derived black liquor. Biores Technol. 2012;111:255-60.

3. Zhang $\mathrm{X}, \mathrm{Xu}$ J, Cheng JJ. Pretreatment of corn stover for sugar production with combined alkaline reagents. Energy Fuels. 2011;25(10):4796-802.

4. $\mathrm{Xu}$ J, et al. Gamagrass varieties as potential feedstock for fermentable sugar production. Biores Technol. 2012;116:540-4.

5. Zhang $X$, et al. Maleic acid and aluminum chloride catalyzed conversion of glucose to 5-(hydroxymethyl) furfural and levulinic acid in aqueous media. Green Chem. 2016;18(19):5219-29.

6. Zhang X, Hewetson BB, Mosier NS. Kinetics of maleic acid and aluminum chloride catalyzed dehydration and degradation of glucose. Energy Fuels. 2015;29(4):2387-93.

7. Xiao W, et al. Rapid liquefaction of corn stover with microwave heating. BioResources. 2015;10(3):4038-47.

8. Qu T, et al. Ball milling for biomass fractionation and pretreatment with aqueous hydroxide solutions. Acs Sustain Chem Eng. 2017:5(9):7733-42.

9. Zhang YHP, et al. Advances in cellulose solvent- and organic solventbased lignocellulose fractionation (COSLIF). Washington: ACS publication; 2010. p. 365-79.

10. Hewetson BB, Zhang X, Mosier NS. Enhanced acid-catalyzed biomass conversion to hydroxymethylfurfural following cellulose solvent-and organic solvent-based lignocellulosic fractionation pretreatment. Energy Fuels. 2016:30:9975-7.

11. Zhao H, Holladay JE, Kwak JH, et al. Inverse temperature-dependent pathway of cellulose decrystallization in trifluoroacetic acid. J Phys Chem B. 2007;111(19):5295-300.

12. Hon DNS, Shiraishi N. Wood and cellulosic chemistry, revised, and expanded. Boca Raton: CRC Press; 2000.

13. Krässig HA. Cellulose: structure, accessibility and reactivity. Philadelphia: Gordon and Breach Science Publ.; 1993.

14. Götze K. Chemiefasern nach dem Viskoseverfahren (Reyon und Zellwolle). Berlin: Springer; 2013. 
15. Shiga TM, et al. Enhanced rates of enzymatic saccharification and catalytic synthesis of biofuel substrates in gelatinized cellulose generated by trifluoroacetic acid. Biotechnol Biofuels. 2017;10(1):310-25.

16. Terinte N, Ibbett R, Schuster KC. Overview on native cellulose and microcrystalline cellulose I structure studied by X-ray diffraction (WAXD): comparison between measurement techniques. Lenzinger Berichte. 2011;89:118-31.

17. Zhang Y-HP, et al. A transition from cellulose swelling to cellulose dissolution by o-phosphoric acid: evidence from enzymatic hydrolysis and supramolecular structure. Biomacromol. 2006;7(2):644-8.

18. Chen $L$, et al. Integrated chemical and multi-scale structural analyses for the processes of acid pretreatment and enzymatic hydrolysis of corn stover. Carbohydr Polym. 2016;141:1-9.

19. Zhang $Y H$, Lynd LR. Determination of the number-average degree of polymerization of cellodextrins and cellulose with application to enzymatic hydrolysis. Biomacromol. 2005;6(3):1510-5.

20. Liu J, et al. The impact of alterations in lignin deposition on cellulose organization of the plant cell wall. Biotechnol Biofuels. 2016;9(1):126-34

21. Nishiyama $Y$, Langan $\neq P, C$ hanzy $\S$. Crystal structure and hydrogenbonding system in cellulose I $\beta$ from synchrotron $X$-ray and neutron fiber diffraction. J Am Chem Soc. 2003;125(47):14300-6.

22. Langan AP, Nishiyama Y, Chanzy $\S \mathrm{H}$. X-ray structure of mercerized cellulose II at $1 \AA$ resolution. Biomacromol. 2001;2(2):410-6.

23. Liu J, et al. Tissue specific specialization of the nanoscale architecture of Arabidopsis. J Struct Biol. 2013;184(2):103-14.
24. Socha AM, et al. Comparison of sugar content for ionic liquid pretreated Douglas-fir woodchips and forestry residues. Biotechnol Biofuels. 2013;6(1):61-6.

25. Larsson PT, Wickholm K, Iversen T. A CP/MAS 13 C NMR investigation of molecular ordering in celluloses. Carbohydr Res. 1997;302(1):19-25.

26. Wickholm K, Larsson PT, Iversen T. Assignment of non-crystalline forms in cellulose I by CP/MAS 13 C NMR spectroscopy. Carbohydr Res. 1998;312(3):123-9.

27. Qu T, et al. Ball milling for biomass fractionation and pretreatment with aqueous hydroxide solutions. ACS Sustain Chem Eng. 2017;5:7733-42.

28. Wormald P, et al. Conversions between ordered and disordered cellulose. Effects of mechanical treatment followed by cyclic wetting and drying. Cellulose. 1996;3(1):141-52.

29. Newman $\mathrm{RH}$. Evidence for assignment of ${ }^{1} 3 \mathrm{C}$ NMR signals to cellulose crystallite surfaces in wood, pulp and isolated celluloses. Holzforschung. 1998;52(2):157-9.

30. Nishiyama Y, et al. Crystal structure and hydrogen bonding system in cellulose la from synchrotron X-ray and neutron fiber diffraction. J Am Chem Soc. 2003;125(47):14300-6.

31. Hall M, et al. Cellulose crystallinity - a key predictor of the enzymatic hydrolysis rate. FEBS J. 2010;277(6):1571-82.

32. Kerley MS, et al. Effect of lignification, cellulose crystallinity and enzyme accessible space on the digestibility of plant cell wall carbohydrates by the ruminant. Food Microstruct. 1988;7(1):59-65.
Ready to submit your research? Choose BMC and benefit from:

- fast, convenient online submission

- thorough peer review by experienced researchers in your field

- rapid publication on acceptance

- support for research data, including large and complex data types

- gold Open Access which fosters wider collaboration and increased citations

- maximum visibility for your research: over 100M website views per year

At BMC, research is always in progress.

Learn more biomedcentral.com/submissions 